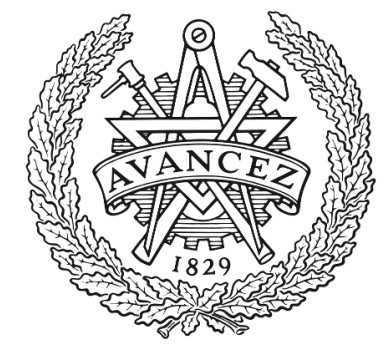

CHALMERS

UNIVERSITY OF TECHNOLOGY

\title{
Static resource allocation for dynamic traffic
}

Downloaded from: https://research.chalmers.se, 2023-04-26 13:30 UTC

Citation for the original published paper (version of record):

Xu, Y., Agrell, E., Brandt Pearce, M. (2019). Static resource allocation for dynamic traffic. IET Conference Publications. http://dx.doi.org/10.1049/cp.2019.1115

N.B. When citing this work, cite the original published paper. 


\title{
Static Resource Allocation for Dynamic Traffic Yuxin $\mathrm{Xu}^{l}$, Erik Agrell, ${ }^{2}$ and Maite Brandt-Pearce ${ }^{I}$
}

\author{
${ }^{1}$ Department of Electrical and Computer Engineering, University of Virginia, USA \\ ${ }^{2}$ Department of Electrical Engineering, Chalmers University of Technology, Sweden \\ yx4vf@virginia.edu,mb-p@virginia.edu
}

Keywords: Optical communications, network optimization, flexible grid networks, random bandwidth traffic, physical layer impairments

\begin{abstract}
A flexible offline probabilistic (FOP) algorithm is designed to aggressively accommodate random bandwidth traffic demands in long-haul networks. Compared to algorithms that configure demands according to their maximum bandwidth, the FOP algorithm can save $15 \%$ of the spectrum used, accommodating over $99 \%$ of the throughput demand.
\end{abstract}

\section{Introduction}

Robust flexible grid optical fiber networks are needed to satisfy growing heterogeneous traffic demands where traffic data-rates can change significantly within hours [1]. Resource planning for continental-scale networks needs to (a) be longterm stable so that existing connections are not disrupted, (b) provide reliable network accessibility, and yet (c) aggressively allocate resources to prevent wasteful over-provisioning.

Offline resource allocation algorithms solve the first two of the requirements stated above, yet severely over-provision available resources. Long-haul networks are modeled as having a slow variability (in terms of months or longer). These so-called static algorithms pre-assign fixed spectrum and routing for all demands. Traffic is configured according to the maximum bandwidth it may need and thus the control plane accommodates all traffic in fixed spectrum slots (referred to as a fixed spectrum assignment) [2].

Online algorithms are dynamic and respond to known changing traffic real-time, or at least within a short time delay (minutes or hours). The control plane may assign resources for one or more time periods, and so the spectrum assignment is said to be flexible [2]. If well designed, these methods satisfy requirements (b) and (c) above. However, dynamic resource reallocation can lead to severe spectrum fragmentation and can disrupt established lightpaths network-wide.

Recent offline algorithms proposed in [2-5] suggest a flexible resource allocation based on online dynamic assignment considering multiple time periods, i.e., allowing time as an extra optimization dimension. In [3,4], a statistical network assignment process (SNAP) algorithm relying on Monte Carlo simulations of randomly selected data-rate demands is used to obtain expected network states. In each simulation trial of a time period, randomly selected demands are optimized as inputs to a static network planning algorithm. Network infrastructure (regeneration nodes) is assigned considering all simulated time periods. For these algorithms, there are so many variables that an astronomically large number of simulation trials would be required to observe all possible network states.

In this paper, we consider a static resource allocation algorithm for time-varying traffic. To avoid disrupting ongoing services, the resources are fixed for a long time (weeks or months), whereas demands can change on a time scale of hours. Such a scenario calls for an entirely new type of resource allocation algorithm, one that can achieve a satisfactory performance over time as the traffic varies. We present the first algorithm of this type and characterize its performance. The proposed algorithm is named the flexible offline probabilistic (FOP) algorithm.

The FOP algorithm models the demands and the spectrum resources needed by these demands in a probabilistic way, and provisions resources based on these probabilistic models so that overall network resources are saved. Since the FOP algorithm does not utilize time-consuming Monte Carlo simulations, its computational complexity is low, on the order of a single simulation trial of SNAP. Compared with standard provisioning (reserving spectrum for the maximum expected traffic bandwidth), our proposed FOP algorithm saves considerable spectrum usage while accommodating almost all of the requested throughput.

Hardware infrastructure elements, such as regeneration nodes, can only be efficiently deployed if a comprehensive offline algorithm, such as the one proposed, is used. Our approach accounts for physical layer impairments (PLIs) and the resulting required regenerator placement using statistical traffic bandwidth information. We propose a probabilistic spectrum Gaussian noise (PSGN) model, based on the standard GN (Gaussian noise) model [6], that can be used in a mixed integer linear programming (MILP) solver to optimally assign regeneration nodes to the network. Through simulation studies, we find that the proposed algorithm saves about a third of the regeneration nodes needed compared with the traditional transmission reach (TR) based PLI model.

In order to save maximum spectrum resources, the FOP 
algorithm assigns spectrum aggressively, allowing the maximum spectrum allocated to adjacent demands to overlap with a low probability of occurrence. This spectrum overlapping is envisioned as manageable for two types of optical networks: sliced spectrum wavelength-division multiplexing (WDM) optical networks [7] and orthogonal frequency-division multiplexing (OFDM) based optical networks [8]. For sliced spectrum WDM networks, multiple independent 2.5-10 Gb/s sub-channels are groomed and transmitted as super-channels of $\geq 40 \mathrm{~Gb} / \mathrm{s}$ [7]. When superchannels collide, overlapping sub-channels are corrupted, but the non-overlapping sub-channels are not affected. For OFDM based optical networks, overlapping subcarriers are corrupted, but the collision will not affect the transmission of the nonoverlapping subcarriers. Systems that share full provisioning information may filter the signals instead of allowing them to overlap, further reducing the effects of allocated-spectrum overlapping.

\section{Flexible Offline Probabilistic Algorithm}

The FOP algorithm comprises three steps, described below and in Algorithm 1. It first step is to route the random-bandwidth demands using the shortest path algorithm and execute a probabilistic spectrum assignment. To account for cross-layer effects, the PSGN model of the PLIs, described below, is then applied after the routing and spectrum assignment. Lastly, an optimal MILP algorithm assigns regeneration nodes globally.

The goal of FOP is to define a frequency range $\left[f_{\text {min }}^{(d)}, f_{\text {max }}^{(d)}\right]$ and a bandwidth distribution within this range for each demand $d$. The cumulative probability function (CDF) of the bandwidth of demand $d$ is assumed given and denoted as $P_{\Delta}^{(d)}(\delta)$, where $\Delta$ is the random bandwidth with realization $\delta \in\left[\delta_{\text {min }}, \delta_{\text {max }}\right]$. For every $\delta$, a starting frequency $f_{\text {start }}^{(d)}(\delta)$ is selected by FOP within the spectrum assigned for $d$, i.e., $f_{\text {start }}^{(d)}(\delta) \in\left[f_{\text {min }}^{(d)}, f_{\max }^{(d)}\right]$. The probability that a frequency $f$ is occupied by demand $d, S^{(d)}(f)$, is shown in Fig. 1. Let $I^{(d)}(f)$ indicate the presence of traffic demand $d$ at frequency $f$; then $\operatorname{Pr}\left[I^{(d)}(f)=1\right]=S^{(d)}(f)$ is demand $d$ 's occupancy distribution.

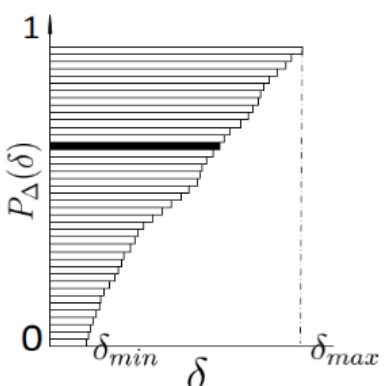

(a)

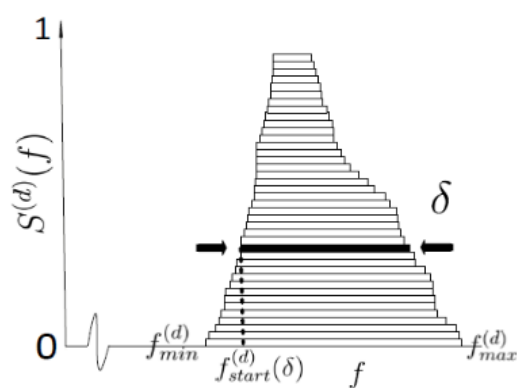

(b)
Fig. 1 (a) CDF of demand $d$ denoted as $P_{\Delta}^{(d)}(\delta)$, and (b) corresponding frequency occupancy distribution $S^{(d)}(f)$.

Often $S^{(d)}(f)$ has long low-probability tails. To maximally preserve as much data as possible without sacrificing spectrum resources and hardware devices, we assign the spectrum aggressively so that there is a small probability $T$ that the spectrum used by adjacent connections overlaps. As mentioned above, for popular candidate optical network architectures, spectrum collisions only affect the overlapped sub-channels (subcarriers) while maintaining the data transmission of non-overlapped sub-channels (subcarriers). The overlapping probability at frequency $f$ is defined as $P_{o l}(f)=\operatorname{Pr}\left[\sum_{d} I^{(d)}(f)>1\right]=1-\prod_{d} \overline{S^{(d)}(f)}-$ $\sum_{d} S^{(d)}(f) \prod_{l \neq d} \overline{S^{(l)}(f)}$. We design the FOP so that $P_{o l}(f) \leq$ $T, \forall f$.

The traditional GN model was not designed to estimate the cross-channel interference for random bandwidth traffic where connections have been provisioned over overlapping spectral resources. Thus, a PSGN is proposed to calculate the average PLIs based on the GN model in a probabilistic way. Define the spectrum occupancy probability at frequency $f$ for all demands as $P_{o c c}(f)=\operatorname{Pr}\left[\sum_{d} I^{(d)}(f)>0\right]=1-\prod_{d}[1-$ $\left.S^{(d)}(f)\right]$. The PSGN computes the expected nonlinear noise on a channel of interest caused by other random bandwidth demands. If $G N^{(d)}(\delta)$ is the noise caused by interfering signal $d$ with bandwidth $\delta$, the PSGN computes $E\left[\sum_{d} G N^{(d)}(\delta)\right]$. It does this by accumulating the noise contributed by a signal at frequency $f$ times $P_{o c c}(f)$.

Lastly, regeneration nodes containing regeneration circuits need to be assigned optimally, which in this paper is done using an MILP algorithm. The optimization objective is the total number of regeneration nodes. Each regeneration circuit serves one lightpath, and an upper bound on the number of circuits per regeneration node is set to $C_{\max }$.

\section{Algorithm 1 Flexible Offline Probabilistic Algorithm \\ Input: time varying demands $\mathbb{D}$; network topology with a set of links $\mathbb{L}$ and nodes $\mathbb{N}$; overlapping threshold $T$ for spectrum points; network parameters $\mathbb{F}$.}

Definitions: $\mathbb{R}$ is the routing for the input demands; $\mathbb{S}$ is the traffic occupancy distribution $\left(S^{(d)}(f) \forall d, f\right)$ on the links $\mathbb{L} ; G N^{(\mathbb{D})}$ is the noise pattern for the demands $\mathbb{D} ; \mathbb{I}$ is the regeneration assignment for the nodes $\mathbb{N}$; $d$ is the index of the current demands, with an initial value $d=1 ; R_{d} \in \mathbb{R}$ is the routing for a demand $d \in \mathbb{D}$

\section{procedure FOP Algorithm $(\mathbb{D}, \mathbb{L}, \mathbb{N}, T, \mathbb{F})$}

1. Route demands $\mathbb{D}$ by the shortest-path algorithm:

Return the demands routing $\mathbb{R}$ for $\mathbb{D}$ while $d \leq|\mathbb{D}|$ do

Based on $R_{d}$, use first-fit to find $S^{(d)}(f)$

satisfying $P_{o l}(f) \leq T, \forall f$. Set $d=d+1$

end while

Return the spectrum assignment $\mathbb{S}$ for $\mathbb{D}$

2. Use the PSGN model to generate noise pattern:

Return the noise pattern $G N^{(\mathbb{D})}$

3. Based on $G N^{(\mathbb{D})}$ and $\mathbb{R}$, use MILP for regeneration nodes assignment:

end procedure

Return the regeneration assignment $\mathbb{I}$

Output: $\mathbb{R}, \mathbb{S}, \mathbb{G}_{\text {noise }}$, and $\mathbb{I}$ 


\section{Simulation Settings and Numerical Results}

We simulate the performance of our algorithm on the NSF-24 network [9]. Demand distributions are based on the statistical population and time-varying data rates provided in $[1,10]$. We consider three realizations for each demand's bandwidth: large, medium, and small (the values of which depend on the population served by each node), with probability $\frac{5}{24}, \frac{12}{24}$, and $\frac{7}{24}$, respectively. We simulate our FOP algorithm with three overlapping thresholds $T=5 \%, 15 \%$, and $30 \%$. The total network throughput is calculated by a path-based algorithm with respect to each demand. We consider two benchmark spectrum allocation methods: standard static provisioning that uses the maximum bandwidth for each demand (equivalent to $T=0 \%$ ), and a provisioning method that accommodates only the mean value of the bandwidth for each demand (independent of $T$ ). The same time-varying demands are used for all algorithms to test the performance. Demands are sorted in descending order of their medium bandwidths; we then provision up to 300 of the largestbandwidth demands (out of the $24 \times 23=552$ node-to-node demands for the NSF-24 network), covering the largest and most significant data-transmissions in the network. In our simulations, all demands use polarization-multiplexed quadrature phase-shift keying with a required SNR $\geq 8.47 \mathrm{~dB}$. $C_{\text {max }}$ is set to 16 circuits per node.

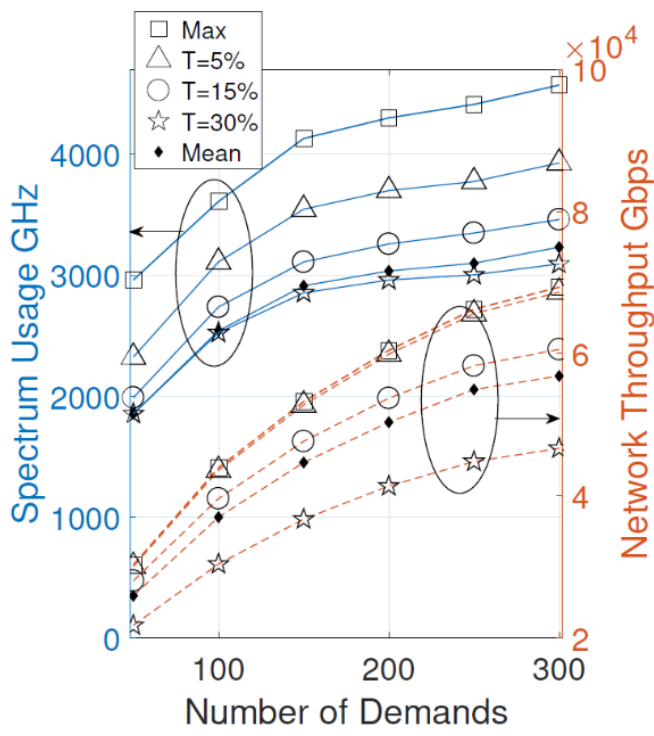

Fig. 2 Trade-off between spectrum usage and throughput as a function of $|\mathbb{D}|$.

Figs. 2 and 3 show the trade-off between spectrum usage, network throughput, and $T$. The FOP algorithm with $T=5 \%$ balances the spectrum usage and the network throughput. Compared to standard planning $(T=0 \%)$, the FOP algorithm with $T=5 \%$ saves $15 \%$ of the spectrum used for 300 demands with only 684 Gbps (less than 1\%) throughput loss out of 68.64 Tbps. When $T$ increases, the spectrum usage and the network throughput both decrease. Compared to FOP with $T=5 \%$, mean-value provisioning saves $29 \%$ of spectrum usage for 300 demands; however, it suffers a $18 \%$ throughput penalty, which is unacceptable.

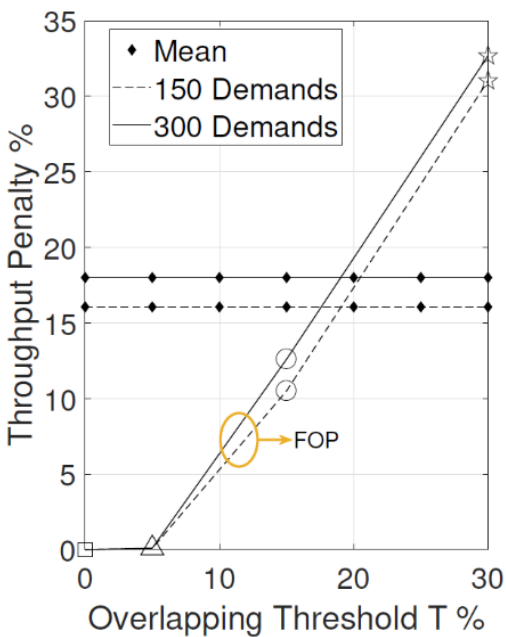

Fig. 3 Throughput penalty as functions of overlapping threshold $T$ for 150 and 300 demands.

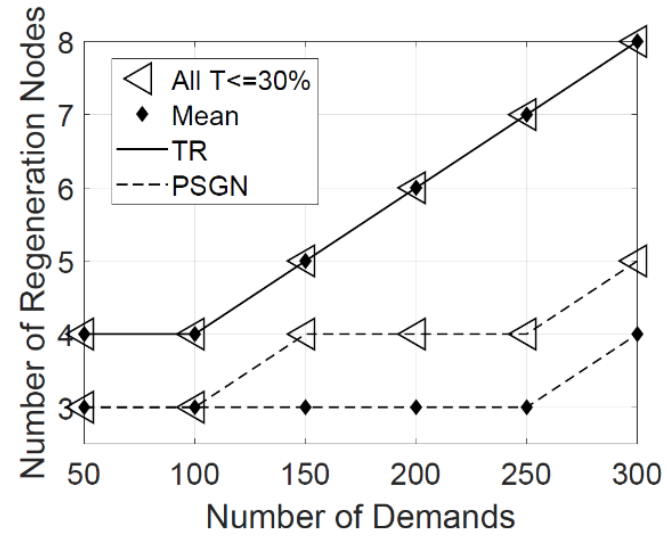

Fig. 4 Number of regeneration nodes needed as functions of $|\mathbb{D}|$ with the TR and PSGN models.

Fig. 4 shows that using the PSGN model, a state-dependent PLI model, compared with a worst-case constraint such as the traditional TR, can significantly reduce the number of regeneration nodes required. The regeneration nodes assignment using the TR model is generated using the same parameters as for the PSGN model in order to make the comparison fair. For 300 demands, the number of regeneration nodes required by PSGN is $37.5 \%$ less than that of the traditional TR. Using PSGN, the number of regeneration nodes required by the mean-value planning is less than that of the other four scenarios. However, applying time-varying bandwidth demands to the planning results (assigned regeneration nodes), the mean-value provisioning algorithm leads to $3 \%$ required-SNR violations due to excessive PLIs (in addition to its $18 \%$ throughput penalty).

\section{Conclusions and Acknowledgements}

The static FOP algorithm is able to accommodate variable bandwidth demands while saving network resources. The FOP algorithm with $T=5 \%$ can provision 300 time-varying demands and save $15 \%$ of the network resources with negligible throughput penalty. In addition, the proposed PSGN model saves $37.5 \%$ in the number of regeneration nodes required compared to the traditional TR model.

This work was supported in part by NSF grant CNS-1718130. 


\section{References}

[1] M. Feknous, T. Houdoin, B. L. Guyader, J. D. Biasio, A. Gravey, and J. A. T. Gijn, "Internet traffic analysis: A case study from two major European operators," in IEEE Symp. Comput. Commun. (ISCC), June 2014.

[2] B. C. Chatterjee, N. Sarma, and E. Oki, "Routing and spectrum allocation in elastic optical networks: A tutorial," Commun. Surveys Tuts., vol. 17, no. 3, pp. 1776-1800, third quarter 2015.

[3] L. Yan, Y. Xu, M. Brandt-Pearce, N. Dharmaweera, and E. Agrell, "Robust regenerator allocation in nonlinear flexiblegrid optical networks with time-varying data rates," J. Opt. Commun. Netw., pp. 823-831, Nov 2018.

[4] M. Cantono, R. Gaudino, and V. Curri, "Potentialities and criticalities of flexible-rate transponders in DWDM networks: A statistical approach," J. Opt. Commun. Netw., pp. A76A85, July 2016.

[5] M. Klinkowski, M. Ruiz, L. Velasco, D. Careglio, V. Lopez, and J. Comellas, "Elastic spectrum allocation for timevarying traffic in flexgrid optical networks," J. Sel. Areas Commun., vol. 31, no. 1, pp. 26-38, Jan 2013.

[6] P. Johannisson and E. Agrell, "Modeling of Nonlinear Signal Distortion in Fiber-Optic Networks," J. Lightwave Technol., vol. 32, no. 23, pp. 4544-4552, Dec 2014.

[7] R. Dutta and G. N. Rouskas, "Traffic grooming in WDM networks: past and future," IEEE Netw., vol. 16, no. 6, pp. 4656, Nov 2002.

[8] O. Gerstel, M. Jinno, A. Lord, and S. Yoo, "Elastic optical networking: A new dawn for the optical layer?" IEEE Commun. Mag., vol. 50, no. 2, pp. 12-20, Feb 2012.

[9] X. Wang, M. Brandt-Pearce, and S. Subramaniam, "Impact of wavelength and modulation conversion on translucent elastic optical networks using MILP," J. Opt. Commun. Netw., pp. 644-655, July 2015.

[10] M. Batayneh, D. A. Schupke, M. Hoffmann, A. Kirstaedter, and B. Mukherjee, "On routing and transmissionrange determination of multi-bit-rate signals over mixed-linerate WDM optical networks for carrier Ethernet," IEEE/ACM Trans. Netw., pp. 1304-1316, Oct 2011. 